\title{
O estudo da performance do sistema fotovoltaico (on grid) da Escola Politécnica de Pernambuco - POLI/UPE
}

The study of the performance of the photovoltaic system (on grid) of the Pernambuco Polytechnic School - POLI / UPE

\section{Nithyane Rayssa Pires Barros ${ }^{1,2}$ (D) orcid.org/0000-0002-4895-9144}

\section{Carlos Frederico Dias Diniz ${ }^{\mathbf{1 , 3}}$ (iD) orcid.org/0000-0002-5370-2255}

Wallysson Klaus Pires Barros ${ }^{1,4}$ (i) orcid.org/0000-0003-2754-2793

\footnotetext{
${ }^{1}$ Escola Politécnica de Pernambuco, Universidade de Pernambuco, Recife, Brasil,

${ }^{2}$ Graduanda em Engenharia Elétrica Eletrotécnica, Escola Politécnica de Pernambuco, Pernambuco, Brasil,

${ }^{3}$ Mestre em Engenharia Elétrica, Universidade Federal de Pernambuco, Pernambuco, Brasil,

${ }^{4}$ Mestre em Engenharia de Sistemas, PPGES, Escola Politécnica de Pernambuco, Universidade de Pernambuco, Pernambuco, Brasil.
}

E-mail do autor principal: Nithyane Rayssa rayssanithyane@gmail.com

\section{Resumo}

O sistema de geração fotovoltaica encontra-se em expansão por ser, em especial, uma fonte limpa e renovável, com baixos custos de operação, além de não emitirem gases de estufa. Partindo deste ponto de vista, o presente artigo visa analisar os níveis de irradiação do sistema fotovoltaico instalado na Universidade de Pernambuco e determinar diretrizes de localização e angulação dos módulos solares, bem como acompanhar a produção de energia elétrica, implantada pela Companhia Energética de Pernambuco (Celpe), através do Programa de Eficiência Energética da ANEEL (PROPEE). Foram instalados 54 módulos, totalizando uma potência de 12,5 kW e inaugurado no dia 19 de setembro de 2017. Ao término desta fase, foi realizado um estudo sobre orientação, inclinação e irradiação das placas, com a finalidade de proporcionar melhorias no rendimento do sistema. Com o auxílio da plataforma computacional AURORA VISION e do software PVsyst, foi possível monitorar a produção e o desempenho energético das placas solares. Como resultados obtidos nesta pesquisa, foi possível disseminar ações de eficiência energética que, junto à instalação da usina fotovoltaica, colaborou com a redução de custos das contas de energia elétrica. Além disso, foi possível obter o estudo dos fatores que afetam a performance da usina e comprovar, portanto, seu desempenho satisfatório.

Palavras-Chave: Geração fotovoltaica; Plataforma Computacional; Produção de energia; Análise.

\section{Abstract}

The photovoltaic generation system is expanding because it is, in particular, a clean and renewable source, with low operating costs, in addition to not emitting greenhouse gases. From this point of view, this article aims to analyze the irradiation levels of the photovoltaic system installed at the University of Pernambuco and determine guidelines for the location and angulation of the solar modules, as well as to monitor the production of electric energy, implemented by the Companhia 
Energética de Pernambuco (Celpe), through ANEEL's Energy Efficiency Program (PROPEE). 54 modules were installed, totaling a power of $12,5 \mathrm{~kW}$ and inaugurated on September 19, 2017. At the end of this phase, a study was conducted on the orientation, inclination and irradiation of the plates, in order to provide improvements in the performance of the system. With the aid of the AURORA VISION computational platform and the PVsyst software, it was possible to monitor the production and energy performance of solar panels. As results obtained in this research, it was possible to disseminate energy efficiency actions that, together with the installation of the photovoltaic plant, collaborated with the reduction of costs of electricity bills. In addition, it was possible to obtain a study of the factors that affect the plant's performance and prove, therefore, its satisfactory performance.

Key-words: Photovoltaic generation; Computational Platform; Production of energy; Analyze.

\section{Introdução}

Paralelo ao desenvolvimento econômico e tecnológico vivenciado no início do século XX com a inserção da industrialização e alta taxa de urbanização, surgiu o consumo excessivo de eletricidade. Segundo uma prospectiva estudada pela Empresa de Pesquisa Energética (EPE), pode-se afirmar que nos próximos 25 anos está previsto um crescimento na busca por eletricidade [1], como indicado na Figura 1.

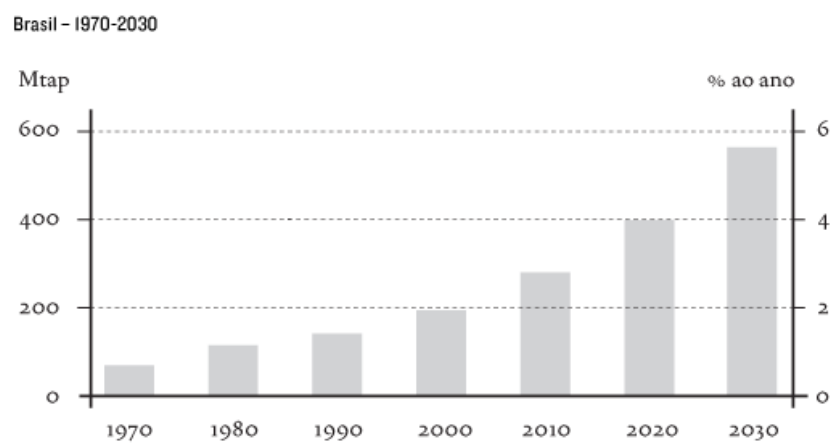

Figura 1: Evolução da demanda de energia e taxa de crescimento econômico. Fonte: Adaptado de EPE (2007).

$\mathrm{O}$ aumento da demanda energética em conjunto com a possibilidade de redução da oferta de combustíveis convencionais e a crescente preocupação com a preservação do meio ambiente estão impulsionando a comunidade científica a pesquisar e desenvolver fontes alternativas de energia menos poluentes, renováveis e que produzam pouco impacto ambiental [2].

De acordo com a Figura 2, é possível verificar que a matriz elétrica brasileira é baseada em fontes renováveis de energia, ao contrário da matriz elétrica mundial. Isso é ótimo para o Brasil, pois além de possuírem menores custos de operação, as usinas, que geram energia a partir de fontes renováveis, contribuem na diminuição das emissões de $\mathrm{CO} 2$ na atmosfera [3].

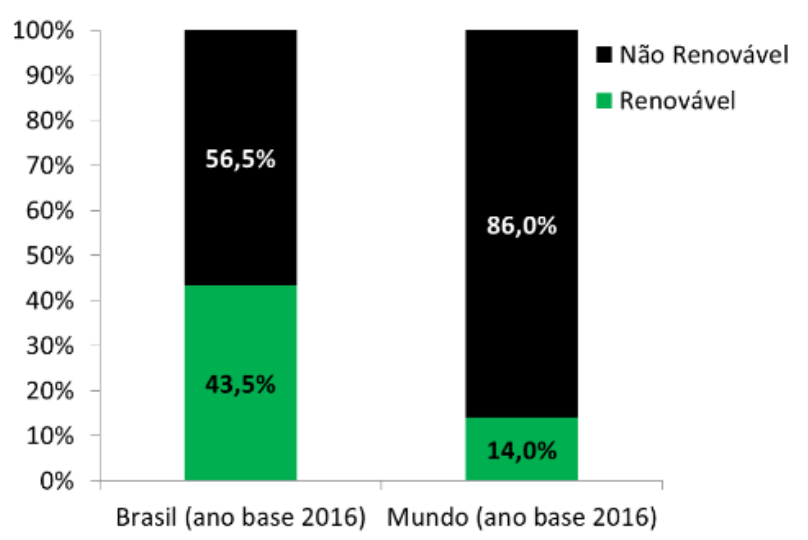

Figura 2: Matriz energética Mundial e Brasileira. Fonte: Adaptado da EPE (2018).

Frente a este contexto, novas fontes estão surgindo para suprir e fortalecer este panorama energético nacional, ou seja, um perfil sustentável. Os primeiros passos para o conceito de sustentabilidade ocorreu em Estocolmo, na Suécia, onde a Conferência Internacional das Nações Unidas sobre o Ambiente Humano (United Nations Conference on the Human Environment), realizada em 1972, ficou conhecida como a Conferência de Estocolmo.

O evento mostrou a incompatibilidade entre desenvolvimento sustentável e os padrões de produção e consumo de energia, trazendo à tona mais uma vez a necessidade de uma nova relação "ser 
humano-meio ambiente". Ao mesmo tempo, teve por objetivo conciliar as questões ambientais, econômicas e sociais, sem trazer a estagnação do crescimento econômico. Em 1987, após dezenas de reuniões da Comissão Mundial sobre o Meio Ambiente e Desenvolvimento, o relatório sobre o Ecodesenvolvimento ficou pronto. Em homenagem à primeira-ministra da Noruega, Gro Harlem Brudtland, indicada pela ONU para chefiar a comissão, o relatório ficou conhecido como Relatório Brundtland.

"Em essência, o desenvolvimento sustentável é um processo de transformação no qual a exploração dos recursos, a direção dos investimentos, a orientação do desenvolvimento tecnológico e a mudança institucional se harmonizam e reforçam o potencial presente e futuro, a fim de atender às necessidades e aspirações humanas" [4], resume o Relatório Brundtland.

Entre as medidas apontadas pelo relatório, constam algumas soluções, como a diminuição do consumo de energia, o desenvolvimento de tecnologias para uso de fontes energéticas renováveis e o aumento da produção industrial nos países nãoindustrializados com base em tecnologias ecologicamente adaptadas.

Segundo a ANEEL, as fontes consideradas renováveis são: solar, eólica, biomassa e hídrica [5]. Dentre as novas fontes de geração, a energia fotovoltaica é a que mais cresce em todo o mundo. 0 seu surgimento veio como uma alternativa de levar a energia para regiões remotas e de difícil acesso. Considerando as informações apresentadas, os primeiros sistemas fotovoltaicos eram isolados da rede elétrica, conhecidos como Sistemas Fotovoltaicos Autônomos (SFA ou off-Grid), ou seja, a energia gerada pelas placas solares alimentava diretamente um banco de baterias que, por sua vez, alimentavam os aparelhos consumidores de energia elétrica.

A utilização do Sistema Fotovoltaico Conectado à Rede (SFCR ou on-grid) surgiu nos anos 90, na Alemanha, caracterizado por ser conectado com a Rede Elétrica da Concessionária. Neste tipo de sistema, a energia gerada é diretamente utilizada pelo consumidor. Nos sistemas on-grid, haverá o fornecimento de energia à concessionária, quando existir a produção de energia elétrica maior que à consumida. A geração fotovoltaica não só possui maior destaque na obtenção sustentável de energia, como também possui uma eficiente aplicação no Brasil, que é um país com alto e constante índice de irradiação solar [6].

\section{Referencial Teórico}

Neste segmento 0 artigo abordará 0 desenvolvimento do tema acerca da performance do sistema fotovoltaico na instituição de ensino em estudo, no que se refere à oferta e demanda por eletricidade e fatores que influenciam no rendimento de tal sistema.

\subsection{A energia solar fotovoltaica}

A energia solar fotovoltaica é originada a partir da conversão direta da luz proveniente do sol em eletricidade. $O$ efeito dessa transformação foi constatado pela primeira vez por Becquerel em 1839, e consiste no aparecimento de uma diferença de potencial numa célula eletroquímica causada pela absorção de luz. Em 1876, um efeito parecido com o constatado por Becquerel foi observado por W. G. Adams e R. E. Day, mas desta vez em um dispositivo em estado sólido fabricado com selênio. C. E. Frits, em 1883, desenvolveu as primeiras células solares de selênio [7].

O baixo rendimento das placas, foi uma das barreiras que impedia o seu rápido desenvolvimento. Porém, a crise do petróleo em 1973, direcionou, mais uma vez, a atenção para o sistema fotovoltaico. Em 1987, a produção da indústria fotovoltaica, no mundo, já passava a marca de 1 Megawatt-pico por ano (MWp/ano) [7], o que evidencia um desenvolvimento substancial neste Mercado.

\subsection{Oferta e demanda de energia elétrica}

O consumo de energia elétrica tem aumentado bastante ao longo dos anos. Na década de 80 era cerca de 6.000 Terawatt-hora (TWh). No século XXI, mais que dobra este valor, como indicado na Figura 3.

DOI: $10.25286 /$ repa.v5i4.1295 


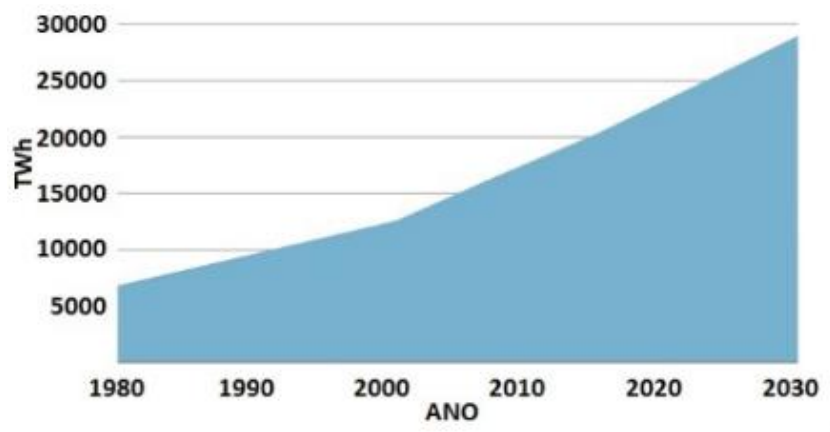

Figura 3: Consumo de energia elétrica nas últimas décadas e previsão até 2030. Fonte: IEA World Energy Outlook (2009).

Paralelo a esta demanda, existe o crescimento da oferta da energia fotovoltaica, não apenas pelo fato do Brasil ter um percentual elevado de insolação durante a maior parte do ano, mas também por existir incentivos fiscais para o incremento desta fonte alternativa de energia. Na Figura 4, é possível ver o quadro de avanço da capacidade instalada dos sistemas fotovoltaicos, representando uma parcela, ainda pequena, de preocupação com a geração de energia limpa e renovável.

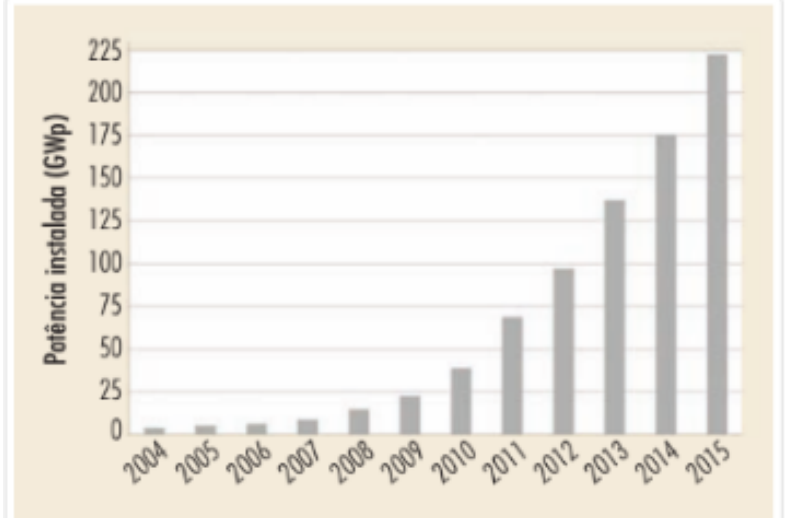

Figura 4: Capacidade instalada global dos sistemas fotovoltaicos. Fonte: Irena (2016).

\section{Estudo de caso}

Diante do cenário de expansão da energia fotovoltaica, apresentado na seção anterior, tem-se a preocupação com a performance dos sistemas, pois o rendimento dos módulos é baixo e, com isso, fatores que interferem na produção devem ser estudados. Vários parâmetros podem afetar o conjunto de módulos solares fotovoltaicos. Eles serão abordados nesta seção.

\subsection{Fatores que afetam a produção de energia solar}

O principal fator que afeta a produção de energia, por meio do sistema fotovoltaico, é o parâmetro de irradiação solar, o qual possui semelhança com o conceito de insolação, porém têm significados diferentes. A irradiação é a intensidade (ou potência) da luz do sol, medida em watts por metro quadrado $\left(\mathrm{W} / \mathrm{m}^{2}\right)$. A potência é um valor instantâneo e, portanto, a irradiação é a intensidade da luz do sol em um determinado momento. Já a insolação (ou radiação) é a quantidade de energia solar que incide em uma superfície durante um certo período de tempo e é medido em Watt-hora ou kilowatt-hora por metro quadrado $\left(\mathrm{Wh} / \mathrm{m}^{2}\right.$ ou $\mathrm{kWh} / \mathrm{m}^{2}$ ). Pelo fato da energia ser expressa em potência ao longo do tempo ( $P \times t)$, a insolação é a irradiação em função do tempo [8].

Assim, primeiramente foi estudada a influência da insolação ao longo do ano para identificação dos períodos de melhor e pior produção da usina fotovoltaica em conjunto com os dias de aulas e férias na instituição. Posteriormente, foi estudada a irradiação no plano inclinado pela SUNDATA, com os cálculos que relatam a melhor angulação das placas. Elas dependem de sua localização geográfica, para adquirir maior rendimento e eficiência, ao se tratar da capacidade que os módulos possuem em converter a luz em eletricidade.

O sombreamento é um outro fator crítico na performance do sistema, pois quando iluminado de forma não homogênea, apresenta resultados significativamente pequenos de produção de energia. Através do estudo de caso na Universidade de Pernambuco, foi visto que este fator não é um agravante, pois os painéis se encontram isolados de chaminés, árvores, postes, antenas ou outros obstáculos que possam proporcionar elevada redução do rendimento do sistema.

Outra causa que demonstra intervenção no rendimento é a inclinação, responsável por auxiliar a autolavagem dos painéis em períodos de chuva, não permitindo o acúmulo de sujeira com o passar do tempo. Desta forma, danificações ao sistema como a deterioração ou oxidação dos painéis não estavam presentes na análise. Uma informação relevante é que 
a usina foi instalada recentemente, oferecendo maior segurança quanto à presença de falhas em circuitos elétricos, apresentando maior produtividade energética, entre outros benefícios.

\subsection{Localização geográfica do sistema}

Inicialmente, foram coletadas informações sobre a localização da Escola Politécnica da Universidade de Pernambuco, que consistiu em estabelecer diretrizes para melhor análise e compreensão da capacidade de geração de energia e de instalação do potencial fotovoltaico. Localizada na Rua Benfica, 455, Madalena - Recife/PE, a POLI/UPE foi uma das cinco instituições escolhidas pela Celpe para receber um dos sistemas de geração fotovoltaica. A diminuição do consumo dessas cinco instituição é equivalente ao consumo mensal de cerca de 150 residências, resultando numa economia de 230 megawatt-hora (MWh) por ano. A Figura 5 mostra o layout do Campus POLI. Destacado em vermelho, tem-se o Bloco $\mathrm{H}$, onde foram instalados os painéis fotovoltaicos (telhado da biblioteca da instituição).

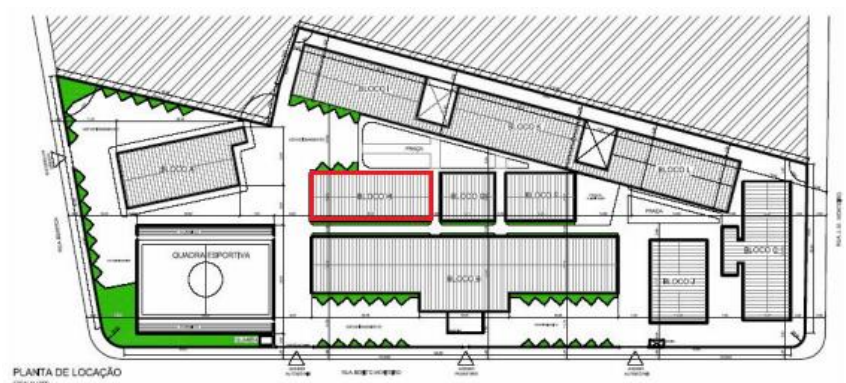

Figura 5: Layout da POLI/UPE. Fonte: POLI/UPE (2013).

A partir das coordenadas geográficas da usina, tornou-se viável a análise de níveis de irradiação e caracterização do sistema fotovoltaico na região. Os dados são mostrados na Tabela 1.

\begin{tabular}{c|c}
\hline Caracterização & Dados \\
\hline Latitude & -8.059 .318 \\
\hline Longitude & -34.903 .474 \\
\hline Elevação & 7.478 .182 \\
\hline
\end{tabular}

Tabela 1: Localização da Usina fotovoltaica. Fonte: Autores (2018).

\section{Metodologia}

O caminho traçado para adquirir os resultados foi por meio de três ferramentas: O programa SUNDATA do CRESESB, a plataforma computacional Aurora Vision da ABB e o software PVsyst. Esta etapa também visa analisar as ações de eficiência energética, que contribuíram para diminuir custos nas contas de energia da instituição.

\subsection{SUNDATA}

O programa disponibiliza, via Web, sugestões para instalação dos painéis fotovoltaicos e possui 17 anos de imagens de satélite com informações de mais de 72.000 pontos em todo o território brasileiro. Assim, suas análises estão embasadas em anos de dados e pesquisas.

Com a busca pelos cálculos da irradiação no plano inclinado, foi possível, de acordo com os dados apresentados no Quadro 1, ter uma base para analisar a melhor angulação das placas solares na localização geográfica em estudo. Ou seja, a partir destas informações, foi plausível conduzir a pesquisa para obter a otimização do sistema instalado, indicando o ângulo ideal para o arranjo da usina.

\begin{tabular}{|l|c|c|c|c|}
\hline \multirow{2}{*}{ Ângulo } & \multirow{2}{*}{ Inclinação } & \multicolumn{3}{|c|}{$\begin{array}{c}\text { Irradiação solar diária média } \\
\text { mensal [kWh/m }\end{array}$} \\
\cline { 3 - 5 } & & Média & Mínima & Máxima \\
\hline $\begin{array}{l}\text { Plano } \\
\text { Horizontal }\end{array}$ & $0^{\circ} \mathrm{N}$ & 5,34 & 3,47 & 7,21 \\
\hline $\begin{array}{l}\text { Ângulo igual } \\
\text { a latitude }\end{array}$ & $8^{\circ} \mathrm{N}$ & 5,35 & 3,94 & 6,76 \\
\hline $\begin{array}{l}\text { Maior média } \\
\text { anual }\end{array}$ & $5^{\circ} \mathrm{N}$ & 5,36 & 3,98 & 6,88 \\
\hline $\begin{array}{l}\text { Maior mínimo } \\
\text { mensal }\end{array}$ & $23^{\circ} \mathrm{N}$ & 5,18 & 4,36 & 6 \\
\hline
\end{tabular}

Quadro 1: Cálculo da irradiação no plano inclinado. Fonte: Adaptado de CRESESB-SUNDATA (2018).

\subsection{Aurora Vision}

"A Plataforma de Gerenciamento de Plantas Aurora Vision é a próxima geração de monitoramento e gerenciamento de plantas fotovoltaicas. Essa é uma solução baseada em nuvem na nuvem, disponível por meio de uma interface de usuário do navegador da Web para os dados da planta fotovoltaica, que fornece acesso altamente interativo e em tempo real aos 


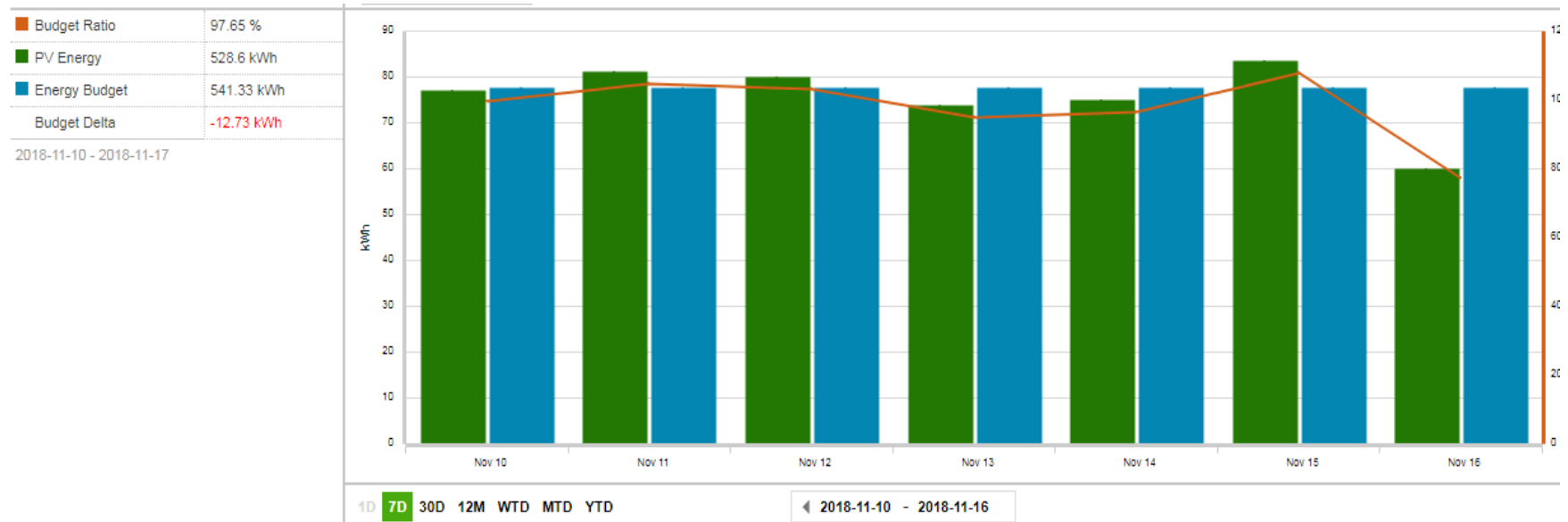

Figura 6: Plataforma Aurora Vision: Monitoramento da produção da usina. Fonte: ABB (2018).

principais indicadores de desempenho e operações para ajudar no acompanhamento dos dados" [9]. Esta ferramenta possibilitou o acompanhamento da performance do sistema durante todo o estudo, alinhando diagnósticos, análises ou alertas e gerenciando eventos, como fatores climáticos, que influenciam na baixa ou alta produção da usina. A Figura 6 mostra o monitoramento de produção energética do sistema fotovoltaico. $\mathrm{Na}$ legenda constam as seguintes informações:

Budget Ratio: é a fração entre a energia gerada e a energia esperada, expressa em \%.

PV Energy: é a energia gerada pelo sistema fotovoltaico, expressa em kWh.

Energy Budget: é a energia esperada mensal dividida pelo número de dias do mês, expressa em kWh.

Budget Delta: é a diferença entre o PV energy e o Energy Budget, expressa em kWh.

\subsection{PVsyst}

O software PVsyst é uma ferramenta computacional desenvolvida pela Universidade de Genebra (Suíça), comercializada pela companhia PVsyst AS. Este instrumento tecnológico permite que engenheiros, pesquisadores e arquitetos trabalhem com diferentes níveis de complexidade e representação para análise de sistemas fotovoltaicos 73 on grid, off grid e pumping [10].

O programa possui uma base de dados de irradiação de 22 localidades na Suíça e de 200 localidades ao redor do mundo, inclusive em Pernambuco. Além disso, apresenta as perdas do sistema fotovoltaico e sua taxa de desempenho, especialmente na utilização de SFCRs. [7]. O PVsyst, em resumo, apresenta relatórios completos com gráficos, tabelas específicas e dados sobre 0 desempenho da usina.

Primeiramente foi realizada a análise dos níveis de insolação in loco. Assim, foi possível identificar pequenas perdas como indicadas nas Figuras 7 e 8 .

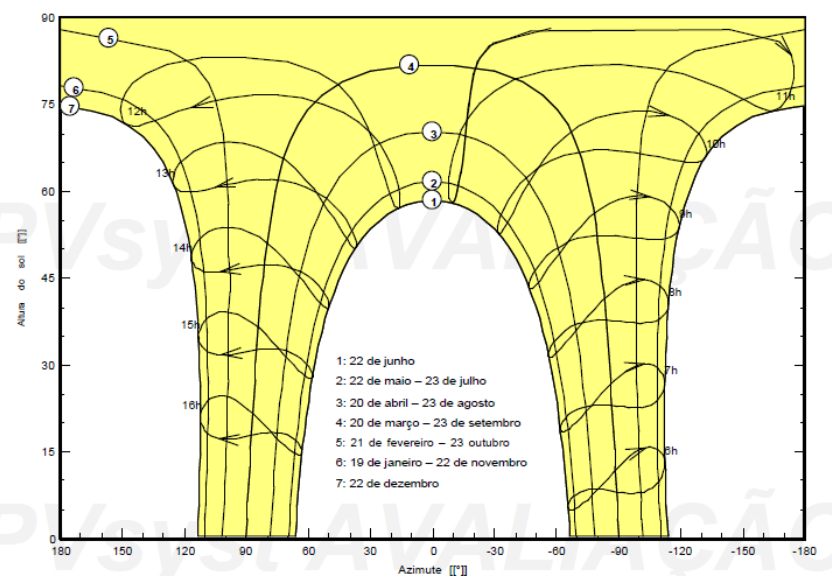

Figura 7: PVsyst - Mapa solar. Fonte: PVsyst (2019). 
Índice de performance (PR)

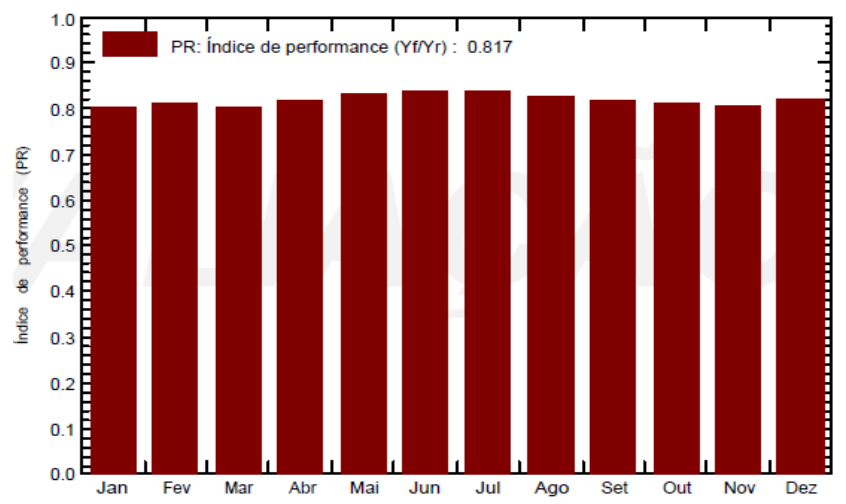

Figura 9: Performance do Sistema Fotovoltaico.

\subsection{Ações de eficiência energética}

A Escola Politécnica da Universidade de Pernambuco foi contemplada para participar do Programa de Eficiência Energética da ANEEL (PROPEE): "O objetivo do programa é promover o uso eficiente da energia elétrica em todos os setores da economia por meio de projetos que demonstrem a importância e a viabilidade econômica de melhoria da eficiência energética de equipamentos, processos e usos finais de energia. Busca-se maximizar os benefícios públicos da energia economizada e da demanda evitada, promovendo a transformação do mercado de eficiência energética, estimulando o desenvolvimento de novas tecnologias e a criação de hábitos e práticas racionais de uso da energia elétrica" [13]. Um conjunto de ações foram desenvolvidas a fim de obter lucro para instituição de ensino, através da utilização da energia elétrica eficiente.

As ações foram divididas em três fases, realizadas de forma respectiva e objetiva, iniciando a primeira e a segunda etapa em janeiro de 2017 e finalizando abril do mesmo ano, e a última etapa foi finalizada em 17 de setembro de 2017, culminando por fim, na inauguração da usina. O processo é demonstrado no Quadro 2. 


\begin{tabular}{|c|c|c|}
\hline ETAPA 1 & ETAPA 2 & ETAPA 3 \\
\hline $\begin{array}{l}\text { - Diagnóstico } \\
\text { energético } \\
\text { POLI/UPE, } \\
\text { seguindo } \\
\text { procedimentos } \\
\text { PROPEE. }\end{array}$ & $\begin{array}{l}\text { - Substituição de } \\
1942 \text { lâmpadas } \\
\text { incandescentes de } \\
40 W \text { para LED"s de } \\
18 \text { e troca de } 24 \\
\text { ar-condicionados } \\
\text { ineficientes, por } \\
\text { novos com selo } \\
\text { PROCEL A. }\end{array}$ & $\begin{array}{lr}\text { - Instalação } & \text { da } \\
\text { Usina Fotovoltaica } \\
\text { conectada à rede, } \\
\text { na } & \text { Escola } \\
\text { Politécnica } & \text { de } \\
\text { Pernambuco. } & \end{array}$ \\
\hline
\end{tabular}

Quadro 2: Fases para a instalação da usina. Fonte: Autores (2018).

\section{Resultados}

Conhecendo os fatores que afetam a produção do sistema e utilizando as ferramentas descritas na seção anterior, foi possível obter ótimos resultados e, consequentemente, estabelecer diretrizes para reparar o arranjo atual com a finalidade de maximizar a produção energética do sistema.

\subsection{Descrição e geração do sistema}

As características técnicas do módulo fotovoltaico e do inversor, utilizados neste SFCR, podem ser conferidas por meio da Figura 10.

\begin{tabular}{|c|c|c|}
\hline CARACTERÍSTICA & Inversor & Módulos \\
\hline Tensão C.C. Máxima & $900 \mathrm{~V}$ & $1000 \mathrm{Vec}$ \\
\hline Faixa de Operação SPMP & $360 \ldots .750 \mathrm{~V}$ & - \\
\hline Corrente C.C.Máxima & $36 \mathrm{~A}$ ISPMP & $8,75 \mathrm{~A}$ \\
\hline Potência Nominal & $12,5 K W(C A)$ & $270 \mathrm{~W}$ \\
\hline Tensão Nominal & $400 \mathrm{Vca}$ & $30,8 \mathrm{Vec}$ \\
\hline Corrente C.A. Máxima & $20 \mathrm{~A}$ & - \\
\hline Fator de Potência & 0,995 & - \\
\hline Temperatura de Operação & $-25^{\circ} \ldots+60^{\circ}$ & - \\
\hline Nי de Strings em Paralelo & 3 & - \\
\hline Tensão em Circuito Aberto & - & 37,9 vec \\
\hline Corrente de Curto-Circuito & - & $9,32 \mathrm{~A},[\mathrm{CC}]$ \\
\hline
\end{tabular}

Figura 10: Características do inversor de frequência e dos módulos. Fonte: Insole (2018).

Analisando as informações expostas da Figura 10, entendeu-se melhor o funcionamento do sistema fotovoltaico, desde a temperatura de operação do inversor de frequência até a máxima corrente que o módulo pode atingir.

Até o dia 11 de novembro de 2018, como mostrado na Figura 11, houve uma geração de $27,76 \mathrm{MWh}$ pelo sistema fotovoltaico, o que resulta numa média de aproximadamente $1.982,85 \mathrm{kWh}$ ao mês. Significa que esta energia foi utilizada pela própria universidade, que é equivalente a cerca de $5 \%$ do consumo total. Assim, foi possível destinar esse lucro a outros investimentos na unidade de ensino.

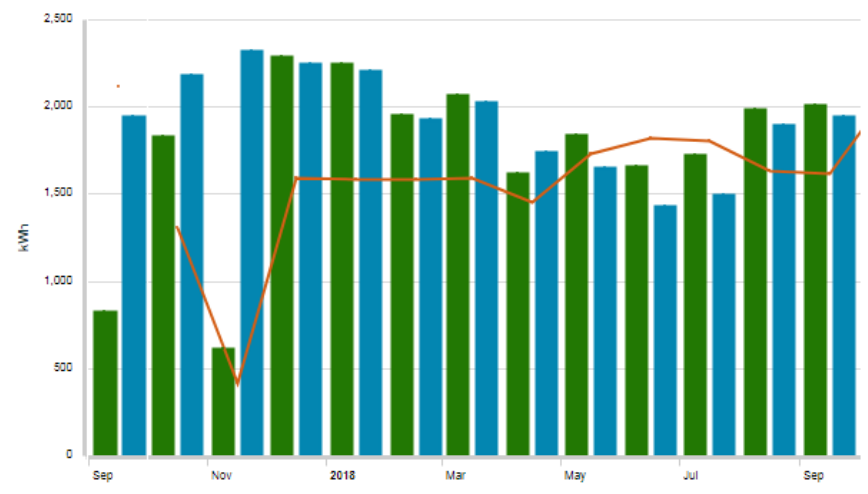

Figura 11: Geração da usina fotovoltaica. Fonte: Aurora Vision (2018).

\subsection{Consumo de energia elétrica da POLI/UPE}

Os benefícios financeiros com as ações de eficiência energética proporcionaram uma redução de $15,24 \%$ no consumo de energia elétrica da unidade, passando de 692,80 MWh para 587,20 MWh anuais. De acordo com o Quadro 3, é verificado qual o percentual total esperado de demanda que a instituição passou a consumir.

\begin{tabular}{|l|l|}
\hline \multicolumn{2}{|c|}{ Resultados esperados } \\
\hline Redução de demanda na ponta total (Kw) & 19,07 \\
\hline Energia Economizada total (MWh/ano) & 105,60 \\
\hline Economia de Energia (\%) & $15,2 \%$ \\
\hline
\end{tabular}

Quadro 3: Diagnóstico energético. Fonte: POLI/UPE (2016).

O custo total do projeto foi de $R \$ 366.851,69$ Reais e espera-se que em sete anos, sem contar os reajustes tarifários, a economia gerada seja suficiente para eficiência. Com a ação conjunta da substituição do sistema existente e a inauguração da usina, levantou- 


\section{Consumo de Energia Total POLI - kWh}

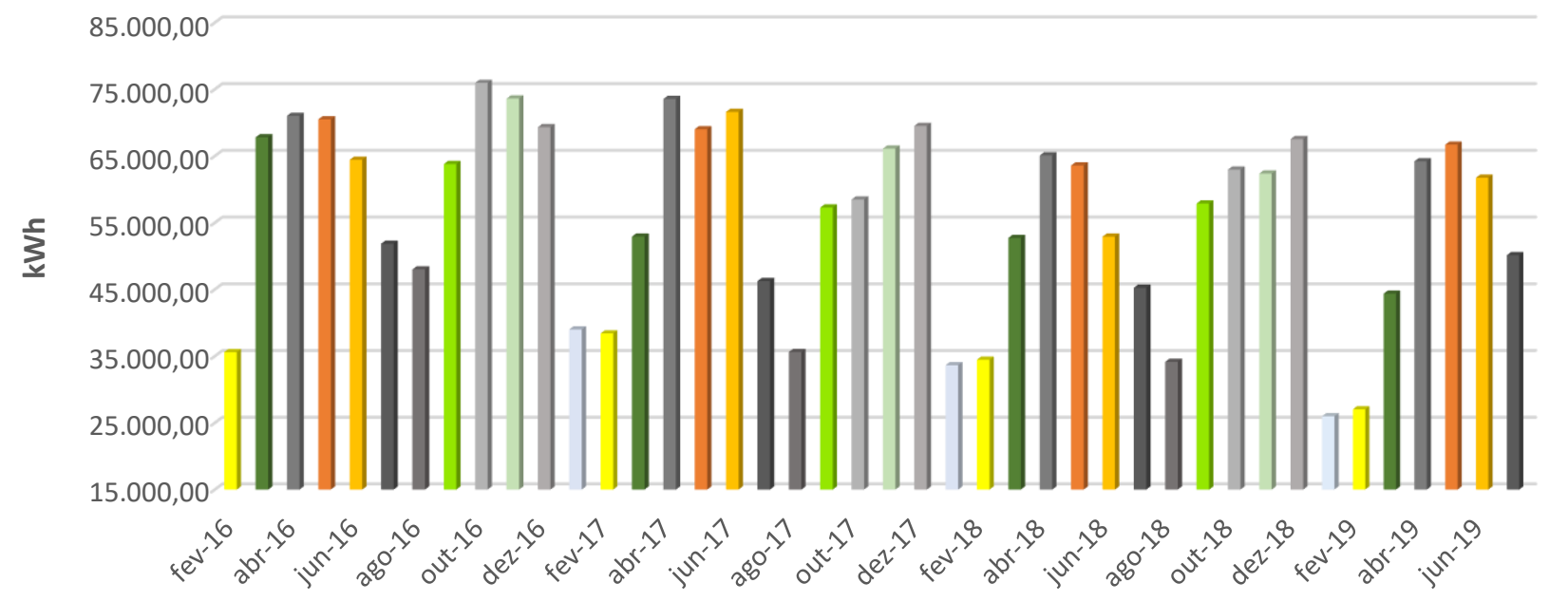

Figura 12: Consumo de Energia Elétrica da POLI/UPE. Fonte: Autores (2018).

se os dados, apresentados na Figura 12 , sobre a conta cobrir o valor total da implantação das ações de mensal da POLI, junto à Celpe, que era, em média, R\$ $40.000,00$ reais, agora está em torno de $\mathrm{R} \$ 35.500,00$ reais, representando uma economia de $\mathrm{R} \$ 4.500,00$ reais mensais, além do menor consumo da energia elétrica, economizando assim, 8,8 MWh/mês ou 105,6 MWh/ano para a instituição.

\subsection{Análise Prática}

Em outubro de 2018, foi realizado uma análise prática do sistema fotovoltaico da POLI/UPE. Foram desenvolvidas ações de medição de angulação e orientação da placa, inspeção da sua integridade e funcionamento, além de avaliar o sombreamento das placas, como mostrado na Figura 13. Todos esses fatores influenciam no rendimento do sistema e foram igualmente avaliados e estudados.

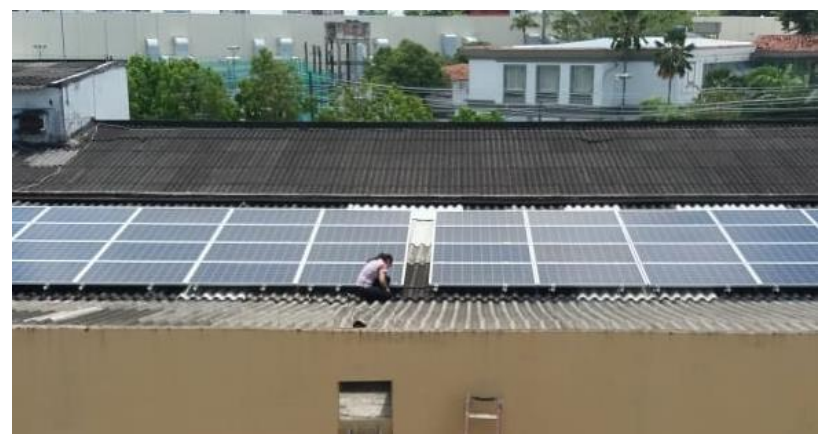

Figura 13: Monitoramento das Placas do sistema fotovoltaico instalado na Universidade de Pernambuco. Fonte: Autores (2018).

A avaliação prática confirmou o estudo teórico desenvolvido, apresentando, portanto, valores adequados de orientação (apontando à direção norte) e inclinação das placas, como por ser visto nas Figuras 14 e 15. 


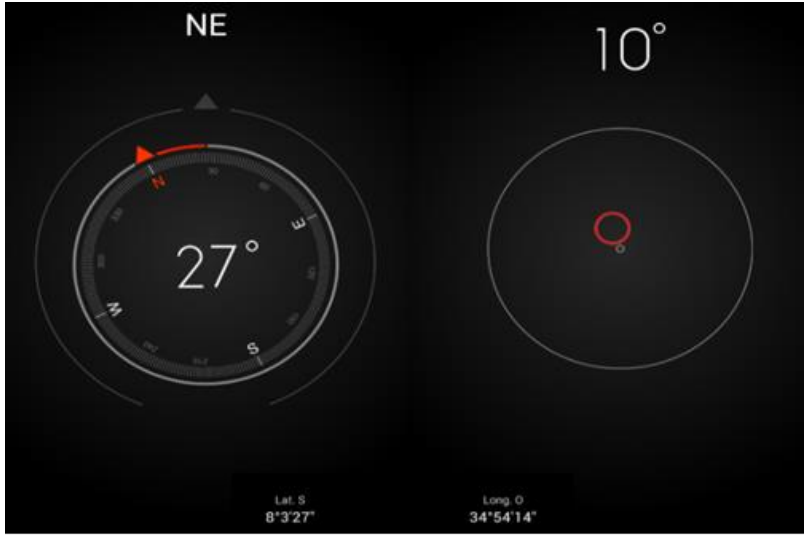

Figura 14: Orientação e inclinação da usina. Fonte: Autores (2018).

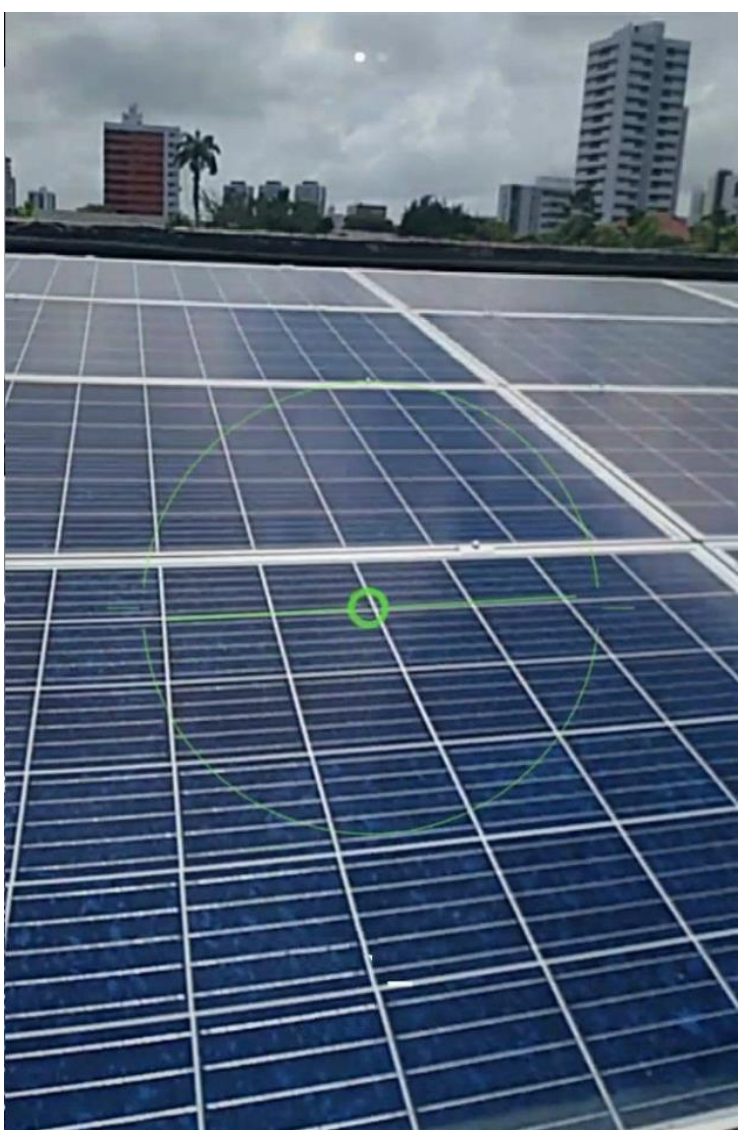

Figura 15: Módulos fotovoltaicos da usina. Fonte: Autores (2018).

\section{Conclusão e sugestões}

A instalação da usina trouxe resultados bastante positivos para a instituição de ensino, pois além de disseminar ações de eficiência energética, colaborou com a redução de custos das contas de energia elétrica.

Após a instalação, iniciou-se o estudo dos fatores que influenciam o rendimento do sistema de geração fotovoltaica. Estabeleceram-se diretrizes com a finalidade de trazer melhorias em sua performance. Logo, o estudo de tais fatores comprovou, por meio do programa SUNDATA, que a localização do sistema proporcionou alta geração de eletricidade, como também não indicou obstrução/danificação do equipamento por ter sido adquirido há pouco tempo.

Os módulos fotovoltaicos têm uma vida útil estimada de mais de 25 anos e degradação da produção, devido ao envelhecimento, em torno 0,7\% ao ano. O fabricante garante que em 10 anos de utilização a potência estará situada entre $90 \%$ e $97 \%$ do valor medido em condições padrão de operação. Após 10 anos (até 25 anos), o fabricante garante que a potência do módulo fotovoltaico será mantida na faixa de $80 \%$ da potência original.

Com o auxílio da ferramenta computacional Aurora Vision foi possível acompanhar em tempo real a produção da usina, alinhando diagnósticos, análises ou alertas e gerenciando eventos. De maneira complementar, tem-se o software PVsyst que analisou por meio de gráficos e mapas solares o ótimo desempenho da usina, comprovando resultados satisfatórios.

Contabilizando o investimento inicial, tem-se uma projeção de retorno financeiro em um período menor que 10 anos. O orçamento poupado poderá ser investido em melhorias para a Universidade.

O estudo futuro, com o auxílio do software PVsize, trará amplificação dos conceitos demonstrados e utilizará de cálculos e teorias para expressar o modo de avaliar e estipular a capacidade e produtividade da usina, além de apresentar gráficos de estado de carga das baterias e tensão ao longo do tempo [7], assim como análises aperfeiçoadas de outras finalidades do sistema.

\section{Referencias}

[1] TOLMASQUIM, Mauricio T. et al. Matriz energética brasileira, uma prospectiva. Novembro, 2007. 
[2] PEREIRA, Enio Bueno et al. Atlas Brasileiro de Energia Solar. $1^{\circ}$. ed. São José dos Campos: [s.n.], 2006. 09 p. Disponível em: <http://ftp.cptec.inpe.br/labren/publ/livros/brazil _solar_atlas_R1.pdf>. Acesso em: 30 jul. 2018.

[3] EPE: Empresa de Pesquisa Energética - Matriz Energética e Elétrica. Disponível em: https://www.epe.gov.br/pt/abcdenergia/ma triz-energetica-e-eletrica. Acesso em: 16 jun. 2020.

[4] COMISSÃO MUNDIAL SOBRE MEIO AMBIENTE E DESENVOLVIMENTO (CMMAD). Nosso futuro comum. Rio de Janeiro: Fundação Getulio Vargas, 1988.

[5] LOPES, Paulo Henrique Silvestre. Nota Técnica no 0043/2010-SRD/ANEEL. Setembro, 2010.

[6] MACÊDO, W.N. et al. Sistemas fotovoltaicos conectados à rede elétrica. São Paulo, Oficina de Textos. 2012.

[7] PINHO, J. T. (Org); GALDINO, M. A. (Org). Manual de Engenharia para Sistemas Fotovoltaicos. 2 ed. Rio de Janeiro, 2014.

[8] Krinat solar: como reduzir a sua conta de energia através da energia solar fotovoltaica. $2017 . \quad$ Disponível em: $<$ https://krinatsolar.com.br/eficiencia-domodulo-fotovoltaico/> Acesso em: 16 out. 2018.

[9] PLANT PORTFOLIO MANAGER-POLI/UPE. 2017. Disponível em: <http://sicessolar.com.br/aurora-visor-plant/>. Acesso em: 16 jul. 2018.

[10] PVSYST - FOTOVOLTAIC SOFTWARE. Disponível em: <https://www.pvsyst.com/>. Acesso em: 23 Mar. 2019.

[11] IEC 61724-1, INTERNATIONAL STANDARD Photovoltaic system performance - Part 1: Monitoring.
[12] SMA. "Performance Ratio. Quality factor for the PV plant", Technical Information, SMA Solar Technology AG, 2018.

[13] Programa de Eficiência Energética. 2016. Disponível em: <http://www.aneel.gov.br/programa-eficienciaenergetica> Acesso em: 10 jun. 2018. 